\title{
Treatment Imprudences for an Adolescent Patient Leading to Esthetic Liability \& Periapical Lesion: A Case Report
}

\author{
Mohammed Zameer ${ }^{1 *}$, Sameen Birajdar ${ }^{2}$, Syed Nahid Basheer ${ }^{3}$ and Naviwala Gulam Anwar \\ ${ }^{1}$ New Al Zahir Dental Clinic, Saada, Salalah, Sultanate of Oman \\ ${ }^{2}$ General Dentist, Maharashtra University of Health Sciences, India \\ ${ }^{3}$ Department of Restorative Dental Science, Jazan University, Kindom of Saudi Arabia \\ ${ }^{4}$ Medical Officer (Dental), Government General Hospital, Malad, Mumbai, India
}

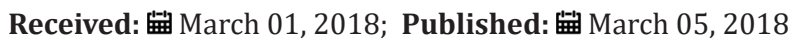

*Corresponding author: Mohammed Zameer, MDS, New Al Zahir Dental Clinic, Saada, Salalah, Sultanate of Oman

\begin{abstract}
Oro-dental injury is an emergency-prompt assessment and appropriate treatment is needed to ensure the best outcome. This case report presents the treatment imprudence's for an adolescent who had suffered from a complicated crown fracture of the maxillary right central incisor that occurred due to a fall, leading to esthetic liability and periapical lesion. Two consecutive treatment stages were defined.

i. Pain management of a non-vital immature tooth through root canal treatment and apexification.

ii. Appropriate full coronal restoration to improve esthetics. This case shows the significance of evaluating pulp vitality and tooth development along with prosthodontic considerations during trauma management for an adolescent to avoid treatment imprudence's. Following appropriate treatment approaches has successfully restored function, esthetics and resolved the periapical pathosis.
\end{abstract}

Keywords: Treatment imprudences; MTA apexification; Adolescent dentistry; Complicated crown fracture

\section{Introduction}

Dental trauma has shown to continue as a significant problem among adolescents which also affects psychologically by unacceptable appearance of damaged teeth [1]. Fracture of enamel, dentin and pulp accounts for $4.8 \%$ of all types of dental injuries among 7-16years of age [2]. In case of trauma to an immature tooth with pulpal exposure, a pulpotomy followed by placement of an appropriate restoration is indicated. Subsequently, when root formation is completed, a definitive restoration can be delivered. Success depends largely on effective management of bacterial contamination at the exposure site and priority to treat the exposed pulp as early as possible. One should not allow chronologic age to preclude performance of whatever treatment is necessary to provide proper function and esthetics. If the teeth involved are fully erupted, have achieved complete root formation, and can be prepared without causing irreversible damage to the pulp, successful prosthodontic treatment can often be provided for patients as young as 12-14years of age [3]. Following case report presents, the treatment imprudence's for an adolescent patient who had suffered from complicated crown fracture leading to esthetic liability, periapical lesion with restricted root formation and their management.

\section{Case Presentation}

A 19year old female reported to New Al zahir Dental Clinic with chief complaint of unaesthetic appearance and pain in previously treated right upper front tooth. She gave a history of trauma 8 years ago, due to fall in house while playing with siblings and the fractured tooth segment was lost. The tooth was asymptomatic but observed tooth discoloration after 14 days of trauma. She was concerned for unaesthetic appearance and discoloration of tooth. Hence, 
undergone treatment by the family dentist, where pulpotomy was performed followed by aesthetic composite restoration. She was not pleased with composite restoration hence; a metal ceramic crown was provided for esthetic satisfaction. She does not recollect any sort of vitality testing done previously. She had observed an increase in gap between crown and gingiva in the first four years after placement of crown, then after its static. She is getting pain in the same tooth since 2 weeks. Clinical examination revealed, red edematous gingiva, accelerated gingival recession with round and thick gingival margin around maxillary right central incisor (Figure 1). Thermal and electric pulp testing revealed non-vitality for maxillary right central incisor. Radiographic investigation revealed an attempt for cervical pulpotomy was made but ended up presenting a periapical lesion having restricted root formation with an open apex (Figure 2).

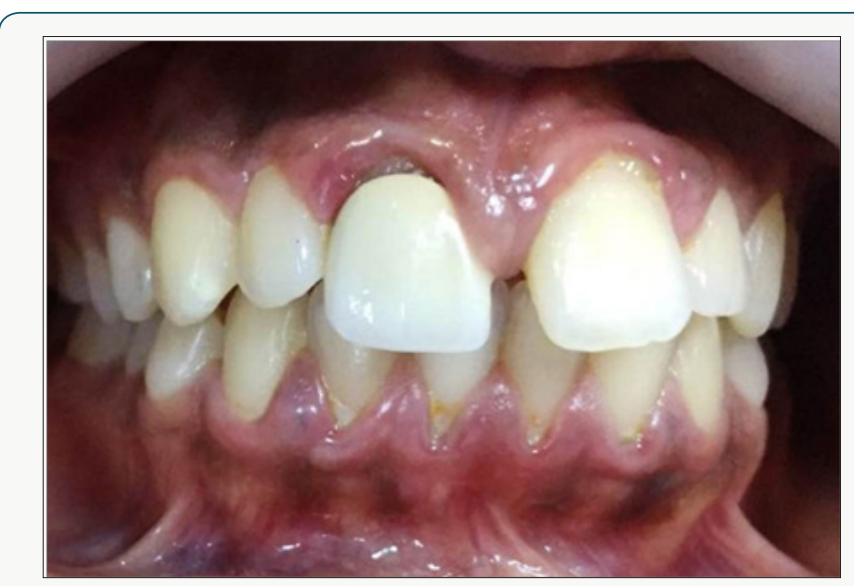

Figure 1.

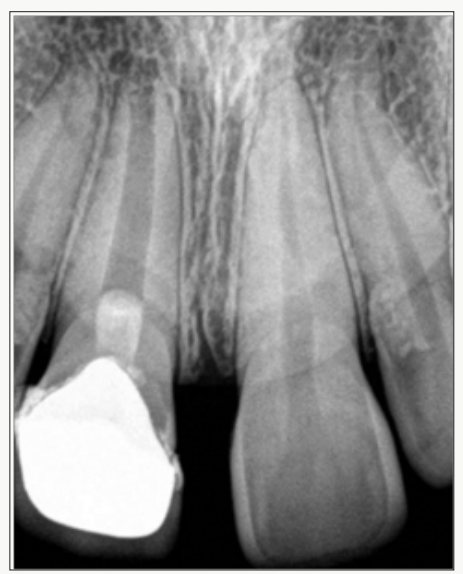

Figure 2.

\section{Clinical Management}

Following are the treatment imprudence's revealed in the presented case.

i. Cervical pulpotomy was performed 14days post trauma for an asymptomatic discolored tooth without taking account of pulp vitality. ii. The traumatized tooth before root formation completion was restored with a metal-ceramic crown.

Management initiated with removal of metal-ceramic crown using a crown remover. Pain management for non-vital immature tooth was done following protocols in accordance to the previous reports through root canal therapy with apexification [4]. As a definitive restoration post endodontic treatment, a metal ceramic crown with a collarless design was given (Figure 3). After 1 year follow-up, gingival inflammation has completely resolved and presented normal form of gingival margin which is thinner and sharper (Figure 4). A periapical radiograph was exposed and it revealed, continuity in lamina dura and consistent width of periodontal ligament space, suggests healing of periapical lesion (Figure 5).

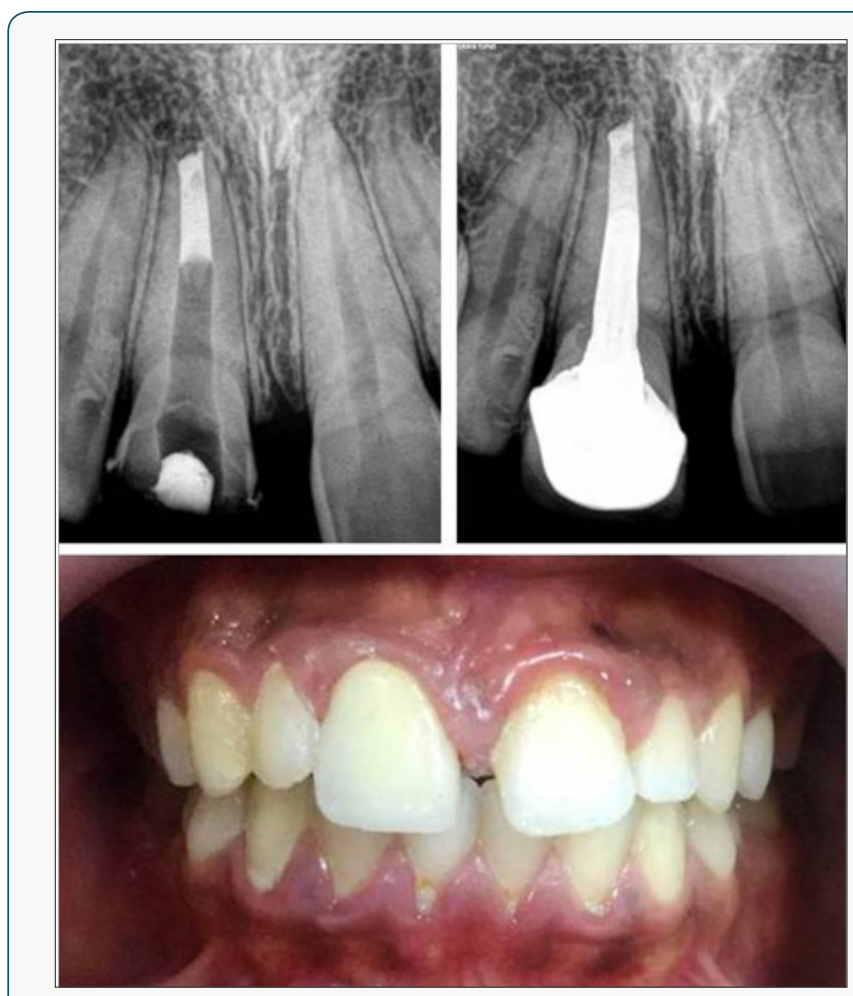

Figure 3.

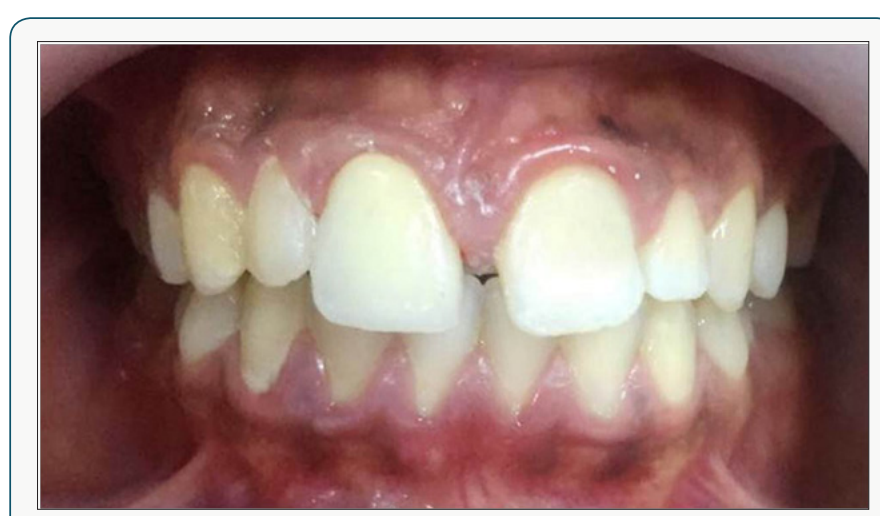

Figure 4. 


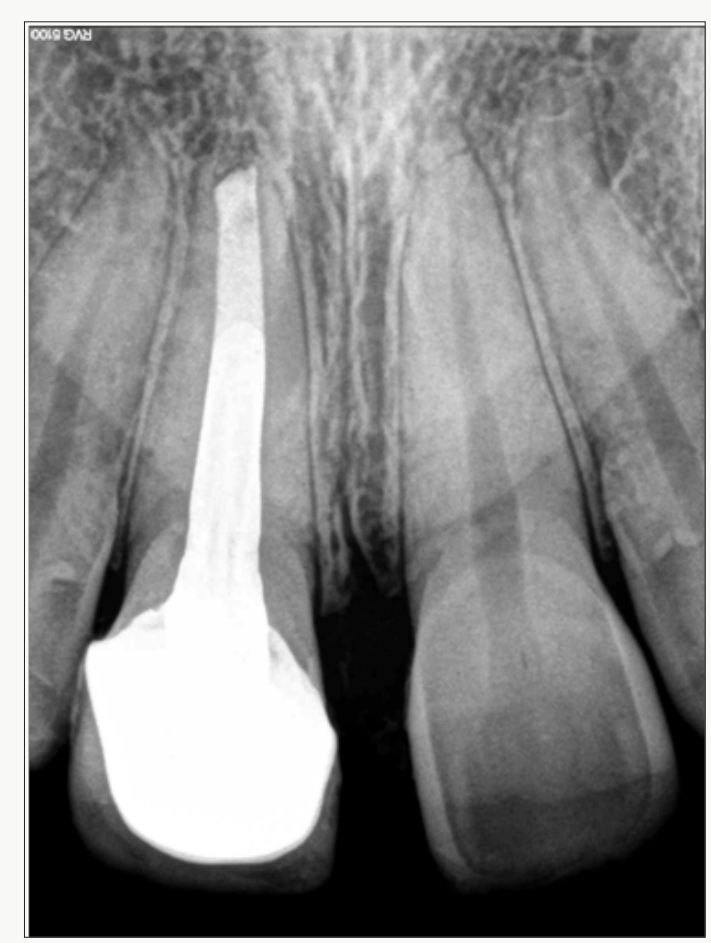

Figure 5.

\section{Discussion}

Oro-dental injury is an emergency-prompt assessment and appropriate treatment is needed to ensure the best outcome. The inconsistency in literature regarding fracture of enamel dentine pulp fracture suggests that, some teeth may develop a hyperplastic reaction and the inflammation remains superficial, while other traumatically exposed pulps may become progressively inflamed and eventually necrotic. Regarding the length of safe interval between trauma and pulpotomy in permanent tooth with complicated crown fracture, the limited information provided in literature suggests that, success will most likely take place when delay in treatment is within 9days [5].

Following are the scientifically reliable interventions available for treating traumatized non-vital immature permanent anterior teeth:

i. Apexification technique;

ii. Regenerative Endodontic Therapy (RET). Recommended best practice based on available evidence, for the management of non-vital immature anterior teeth suggest, use of $\mathrm{Ca}(\mathrm{OH}) 2$ for the traditional apexification technique is no longer advocated as the treatment of choice for such teeth. Instead, it is recommended to use $\mathrm{Ca}(\mathrm{OH}) 2$ for short period of time to achieve disinfection. This should be followed by MTA apexification and obturation of the root canal space [6]. Based on the recommendation, MTA apexification technique was followed in the presented case for the management of non-vital immature tooth. Clinicians may consider using regenerative endodontic therapy in cases where the root development is very incomplete with insufficient amount of dentine, and where it is considered that the tooth has a hopeless prognosis even with application of MTA. In these cases it would be advantageous to gain some deposition of hard tissues through a regenerative approach. However, this is based on weak evidence [6].

Literature suggests when ideal tooth preparation form is compromised; use of strong metal ceramic crown is indicated. Meanwhile, to optimize the cervical esthetics, a metal ceramic crown with collarless design is advised [3]. Whenever possible, cervical margins should not be extended into the gingival sulcus of an adolescent patient. If oral hygiene is inadequate, subgingival margins may produce accelerated gingival recession or interfere with the normal cervical relocation of the gingival tissues as the patient matures. Both occurrences produce an esthetic liability [3]. In the presented case of an immature tooth, a metal ceramic crown was given post pulpotomy with the gingival margin extended subgingivally. Moreover, oral hygiene was poor, that ultimately has lead to an esthetic liability. However, this was managed by providing a metal ceramic crown with a collarless design to optimize esthetics.

\section{Conclusion}

Fracture of enamel-dentin-pulp should promptly be assessed and appropriately treated to ensure best outcome. This case shows the significance of evaluating pulp vitality and tooth development along with prosthodontic considerations during trauma management for an adolescent to avoid treatment imprudence's. Following appropriate treatment approaches have successfully restored function, esthetics and resolved the periapical pathosis.

\section{Acknowledgement}

We would like to extend our heartfelt gratitude towards the management of New Al Zahir Dental Clinic, Saada, Salalah, Sultanate of Oman for their kind support.

\section{References}

1. Gift HC, Bhat M (1993) Dental visits for orofacial injury: defining the dentist's role. Journal of the American Dental Association 124(11): 9298.

2. Roberts G, Longhurst P (1996) Oral and dental trauma in children and adolescents, Oxford University Press Inc, New York, USA.

3. RE McDonald, DR Avery, JA Dean (2004) Dentistry for the Child and Adolescent, Mosby, $8^{\text {th }}$ edition.

4. Kumar V, Zameer M, Prasad V, Mahantesh T (2014) Boon of MTA apexification in young permanent posterior teeth. Case Reports in Dentistry 2014(2014): 673127.

5. Bimstein E, Rotstein I (2016) Cvek Pulpotomy - Revisited. Dental Traumatology 32(6): 438-442.

6. Duggal M, Tong HJ, Al Ansary M, Twati W, Day PF, et al. (2017) Interventions for the endodontic management of non-vital traumatised immature permanent anterior teeth in children and adolescents: a systematic review of the evidence and guidelines of the European Academy of Paediatric Dentistry. European Archives of Paediatric Dentistry 18(3): 139-151. 
(C) (P) This work is licensed under Creative

To Submit Your Article Click Here: Submit Article

DOI: 10.32474/MADOHC.2018.01.000116

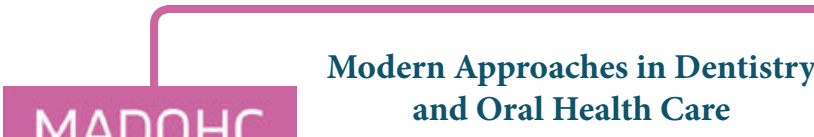

\section{Assets of Publishing with us}

- Global archiving of articles

- Immediate, unrestricted online access

- Rigorous Peer Review Process

- Authors Retain Copyrights

- Unique DOI for all articles 